\section{SOBRE LA VIABILIDAD DE LA INTRODUCCIÓN DE LA VACUNA INACTIVADA CONTRA LA POLIOMIELITIS EN COLOMBIA}

Hemos leído con gran interés el artículo titulado "Impacto económico de la introducción de la vacuna inactivada inyectable contra la poliomielitis en Colombia", publicado por Nelson Alvis y colaboradores en el volumen 27, número 5, de esa Revista (1). Trata sin dudas un tema de gran relevancia para las políticas de vacunación en los países en desarrollo: la viabilidad de sustituir la forma oral de la vacuna contra la poliomielitis (VOP) por la vacuna basada en la forma inactivada del poliovirus (VIP).

Aun cuando el trabajo expone con claridad su objetivo y sus conclusiones, y sus métodos son los usualmente utilizados en estudios de este tipo, creemos que adolece de problemas metodológicos que ameritan una mayor discusión dada la importancia que entraña este problema de salud pública. Nuestras objeciones conciernen fundamentalmente a dos aspectos: la pertinencia de la pregunta propuesta y la validez de los datos que usan los autores para darle respuesta.

Respecto del primer punto, el estudio analiza la mera sustitución de la VOP por la VIP, sin considerar (a excepción de una breve referencia en la discusión) la introducción de vacunas combinadas que incluyan la VIP. En el contexto actual, la posible modificación de las políticas de vacunación en diferentes países pasa por la evaluación del valor agregado de pasar de la VOP a una vacuna combinada que incluya la VIP, puesto que de esta forma se logran obvios beneficios operacionales que no se obtendrían con la VIP sola y que no fueron incluidos en el trabajo de Alvis y colaboradores (1).

En cuanto a los problemas de identificación y validez de los datos utilizados, solo a título ilustrativo mencionaremos que la primera frase del artículo refiere al número de casos de poliomielitis en 2006 utilizando tres citas de las cuales la más reciente es de 2004. Dicho esto, a continuación señalamos las falencias que consideramos más importantes para la obtención de las conclusiones en ese trabajo:

- La revisión de artículos publicados en que se apoyan los autores no cumple con las directivas internacionalmente aceptadas (p. ej. Cochrane [2]). Esto podría ser aceptable puesto que no pretenden realizar una revisión sistemática, pero resulta sorprendente que se intente identificar trabajos sobre costoefectividad sin utilizar ninguno de los términos de búsqueda comúnmente empleados en estudios de este tipo. A raíz de esta falla metodológica, los autores encontraron solamente tres estudios que concluyen que la introducción de la VIP no es costoefectiva (3-5), pero no identificaron dos trabajos que llegan a conclusiones contrarias (ambos aparecen entre los 10 primeros resultados de una búsqueda en Pubmed con los términos "cost-effectiveness inactivated polio"') $(6,7)$.

- No se hace una descripción detallada de los parámetros utilizados en la evaluación económica que permita la replicación de los resultados. Por ejemplo, varias de las referencias utilizadas son inaccesibles o no documentan las afirmaciones de los autores, entre otras las referencias 17 y 34, que deberían dar acceso a los datos utilizados para los cálculos, son inaccesibles usando el enlace publicado e incluso buscándolas en los sitios correspondientes por el título indicado en la referencia.

- El precio utilizado en el artículo para la VIP monovalente (US\$ 7,5) correspondería al de una vacuna combinada con la VIP, ya que el precio de la VIP monovalente es bastante inferior, diferencia que obviamente impacta en los costos calculados para esa sustitución.

- En la discusión, los autores hacen una valoración rápida y pobremente documentada acerca de las vacunas combinadas con la VIP. La afirmación "este beneficio ... [el de la vacuna acelular contra la tos ferina] ... no ha sido comprobado en estudios controlados" es discutible en función de la numerosa evidencia disponible acerca de la existencia de dicho beneficio (8). También es cuestionable la aserción "la vacuna acelular podría interferir con la respuesta inmunológica a otras vacunas, especialmente las que contienen el antígeno vacunal del Haemophilus influenzae tipo $b^{\prime \prime}$. Si bien esa interferencia resulta observable en las vacunas combinadas, no es clínicamente relevante según los expertos, algo que además ha sido demostrado en sistemas de vigilancia epidemiológica nacionales $(9,10)$.

En conclusión, creemos que la evaluación del costo-efectividad de la introducción de la VIP, una medida directamente vinculada a un asunto de salud pública tan sensible como la iniciativa mundial para la erradicación de la poliomielitis, merecería un tratamiento más cuidadoso y mejor documentado. Por ello consideramos perentorio llevar a cabo una evaluación rigurosa, que genere resultados (cualesquiera sean) que permitan a las autoridades de salud pública adoptar decisiones basadas en la mejor evidencia disponible actualmente, a efectos de poder brindar a nuestras sociedades la solución más adecuada. 
Fabián P. Álvarez

Sanofi Pasteur, Lyon, France

Correo electrónico: fabian.alvarez@sanofipasteur.com

José Alejandro Mojica

Sanofi Pasteur, Bogotá, Colombia

Carlos Espinal

Sanofi Pasteur, Washington DC, Estados Unidos

Roberto Debbag

Sanofi Pasteur, Buenos Aires, Argentina

\section{Referencias}

1. Alvis N, de la Hoz F, Narváez J. Impacto económico de la introducción de la vacuna inactivada inyectable contra la poliomielitis en Colombia. Rev Panam Salud Publica. 2010; 27(5):352-9.

2. Higgins JPT, Green S. Handbook for systematic reviewers of interventions. The Cochrane collaboration (2009). Disponible en: http://www.cochrane-handbook.org/. Acceso el 02 de abril de 2011.

3. Miller MA, Sutter RW, Strebel PM, Hadler SC. Cost-effectiveness of incorporating inactivated poliovirus vaccine into the routine childhood immunization schedule. JAMA. 1996;276 (12):967-71.

4. Tucker AW, Isaacs D, Burgess M. Cost-effectiveness analysis of changing from live oral poliovirus vaccine to inactivated poliovirus vaccine in Australia. Aust N Z J Public Health. 2001:25(5):411-6.

5. Griffiths UK, Botham L, Schoub BD. The cost-effectiveness of alternative polio immunization policies in South Africa. Vaccine. 2006;24(29-30):5670-8.

6. Khan MM. Economics of polio vaccination in the posteradication era: should OPV-using countries adopt IPV? Vaccine. 2008;26(16):2034-40.

7. Mascareñas A, Salinas J, Tasset-Tisseau A, Mascareñas C, Khan MM. Polio immunization policy in Mexico: economic assessment of current practice and future alternatives. Public Health. 2005;119(6):542-9.

8. Decker MD, Edwards KM, Steinhoff MC, Rennels MB, Pichichero ME, Englund JA, et al. Comparison of 13 acellular pertussis vaccines: adverse reactions. Pediatrics. 1995;96 (3 Pt 2):557-66

9. Eskola J, Ward J, Dagan R, Goldblatt D, Zepp F, Siegrist CA. Combined vaccination of Haemophilus influenzae type b conjugate and diphtheria-tetanus-pertussis containing acellular pertussis. Lancet. 1999;354(9195):2063-8.

10. Kalies H, Verstraeten T, Grote V, Meyer N, Siedler A, Schmitt $\mathrm{HJ}$, et al; für seltene pädiatrische Erkrankungen in Deutschland Study Group. Four and one-half-year follow-up of the effectiveness of diphtheria-tetanus toxoids-acellular pertussis/Haemophilus influenzae type $\mathrm{b}$ and diphtheria-tetanus toxoids-acellular pertussis-inactivated poliovirus $/ H$. influenzae type $\mathrm{b}$ combination vaccines in Germany. Pediatr Infect Dis J. 2004;23(10):944-50.

\section{RESPUESTA DE AUTOR}

En referencia a la Carta al Editor de Álvarez y colaboradores titulada "Sobre la viabilidad de la introducción de la vacuna inactivada contra la poliomielitis en Colombia", deseamos antes que nada valorar como muy positivo el interés que estos investigadores han mostrado en la evaluación de nuestro artículo (1). De igual modo, nos complace la oportunidad de poder abordar sus inquietudes y comentarios en los siguientes apartes:

1. En primer lugar, los autores de la carta sugieren incluir en el análisis el beneficio agregado de otros biológicos en una vacuna combinada con la forma inactivada del poliovirus (VIP). Tal idea podría ser razonable en países donde no existen las otras vacunas que se combinan con VIP, pero ese no es el caso para Colombia, donde las vacunas contra la hepatitis B y Haemophilus influenzae tipo b han formado parte del Programa Ampliado de Inmunización (PAI) desde hace años. El único beneficio adicional que sí podría agregar sería el cambio de la DPT (difteria, pertussis y tétanos) celular a acelular, el cual si bien no se incluyó directamente en el modelo, sí se analiza en un aparte de la discusión (1). La revisión de la literatura muestra que existen pocos estudios que comparan la eficacia clínica de las vacunas celular y acelular contra pertussis y los resultados son, por lo menos, controversiales. En 1998 Heinninger y colaboradores publicaron un ensayo clínico ( $n=10721$ niños) donde la vacuna celular tenía un efecto protector mayor que la acelular contra la diseminación de la infección en contactos cercanos de los casos primarios ( $84 \%$ vs. $58 \%$ ) (2). En 1996, Greco y colaboradores habían publicado otro ensayo clínico ( $n=14751$ niños) en el cual, contrariamente, hallaron una mayor efectividad en la vacuna acelular que en la celular contra la enfermedad clínica ( $84 \%$ vs. $36 \%$ ) (3). Un estudio de casos y controles en 2005 de Bisgard y colaboradores, por otro lado, no encontró diferencia alguna en la protección clínica entre los dos tipos de vacunas (4). Por ello, supusimos que no había evidencia concluyente respecto a que la efectividad clínica de la DPT celular fuera menor que la demostrada por la acelular. Otros autores han reportado que las dos vacunas solo diferirían en la incidencia de episodios convulsivos después de la vacunación, lo que es todavía materia de debate $(5,6)$. En tal sentido, decidimos no incluir los potenciales efectos benéficos adicionales de introducir la VIP como vacuna combinada en el modelo de análisis.

2. La carta también cita dos artículos que presentarían evidencia contraria a los hallazgos de nuestro trabajo. En realidad, dado que ninguno de estos artículos evalúa el costo-efectividad de la vacuna de polio inyectable, sus resultados no pueden ser interpretados como evidencia contraria. El trabajo de Mascareñas y colaboradores es esencialmente sobre microcosteo de una jornada de vacunación de una semana en México, donde el beneficio analizado es el costo por niño vacunado y no el costo de disminuir la carga de enfermedad, que fue el beneficio analizado en nuestro estudio (7). El segundo estudio - de Khan- mencionado es un análisis ecológico que estima lo que pasaría en forma agregada en 148 países, con diferentes niveles de ingreso, si se tomara la decisión de reemplazar la forma oral de la vacuna contra la poliomielitis (VOP) por la VIP (8). 
Lo que analiza nuestro trabajo, en cambio, son los datos para un país específico de ingreso medio bajo y con precios de la vacuna muy diferentes (1).

3. Khan usa un rango de precios de entre US\$ 0,1 y US\$ 5 para la VOP y de US\$ 1,0 a US\$ 9,0 para la VIP. Como se puede ver, la superposición de rangos podría inducir a errores en la interpretación del resultado. En nuestro estudio se consideran precios de US\$ 0,5 a 1,5 para VOP y de US\$ 5,0 a 7,5 para VIP, rangos que no se sobreponen y que por lo tanto permiten una mejor interpretación.

4. Es necesario resaltar que los resultados de los estudios de costo-efectividad pueden variar de país a país y muchas veces no son extrapolables entre países por las diferencias de costos de enfermedad o de intervenciones. Hay abundante literatura que analiza la transferibilidad o generalizabilidad de los resultados en las evaluaciones económicas de tecnologías sanitarias (EETS) (9-12).

5. Todos los parámetros clave para ejecutar el modelo aparecen en el cuadro 1 de nuestro artículo (1). Además, el cuadro 2 incluye el riesgo estimado de poliomielitis paralítica en diversos países y regiones de manera detallada. Adicionalmente, se han verificado los enlaces electrónicos de las referencias 17 y 34 y, aunque la referencia 34 continúa funcionando sin ningún problema, efectivamente el enlace electrónico de la referencia 17 ya no está activo por razones que desconocemos. Sin embargo, desde febrero de 2011 la referencia 17 podía accederse en: http://www.minproteccionsocial.gov.co/salud/ Paginas/pai.aspx.

6. En el análisis de sensibilidad de nuestro trabajo se considera un costo mínimo de US $\$ 5,0$ para la VIP, pero aun así esa intervención no es costo-efectiva (1). Como ya se señaló, por razones logísticas no es valioso considerar la opción de vacuna monovalente ya que las coberturas de vacunación muy probablemente descenderían.

7. Concordamos con Álvarez y colaboradores en que no realizamos una revisión sistemática como fue planteado en los métodos del artículo. Sin embargo nuestra revisión de literatura fue amplia, con criterios de búsqueda claros y con criterios de inclusión y exclusión verificables. Aunque no podemos asegurar que no exista literatura gris sobre el tema en Latinoamérica que pudo haber quedado por fuera del análisis, tampoco hay razones para pensar que esos hipotéticos trabajos cambien de manera radical nuestras conclusiones.

Una aclaración adicional es que nuestro estudio fue hecho desde la perspectiva de un ministerio de salud y que, por tanto, solo incluyó los beneficios relevantes de las estrategias comparadas, sin incluir potenciales beneficios que todavía están en discusión. En tal sentido, creemos que la evaluación del costoefectividad de la introducción de la VIP en Colombia es "cuidadosa, bien documentada y rigurosa" y discute los resultados de manera responsable usando estudios con los cuales son comparables (13-15).
En conclusión, no encontramos que las objeciones señaladas por Álvarez y colaboradores a los resultados de nuestro estudio pongan en riesgo su validez, ya que en el análisis de sensibilidad se tuvieron en cuenta variaciones importantes de los parámetros más críticos, como lo son el costo de la vacuna y la tasa de enfermedad paralítica asociada a virus vacunal. Aun cuando se usaron rangos extremos para esas variables, la conclusión seguía siendo similar: el cambio de la vacuna oral a inyectable no es costo-efectivo para el nivel de ingreso colombiano a los precios actuales de la vacuna VIP en su presentación combinada. Sin embargo, al final de la discusión reconocimos que la decisión sobre realizar el cambio no depende solo de criterios de costo-efectividad: "La decisión, entonces, debe basarse en consideraciones de tipo ético, económico (por el presupuesto del PAI) y de prioridades de la salud pública nacional" (1).

Nelson Alvis

Universidad de Cartagena, Departamento de Investigaciones Económicas y Sociales

Bolívar, Colombia

Correo electrónico: nalvis@yahoo.com

Fernando de la $\mathrm{Hoz}$

Universidad Nacional de Colombia,

Facultad de Medicina

Bogotá, Colombia

Javier Narváez

Universidad Nacional de Colombia, Facultad de Medicina

Bogotá, Colombia

\section{Referencias}

1. Alvis N, de la Hoz F, Narváez J. Impacto económico de la introducción de la vacuna inactivada inyectable contra la poliomielitis en Colombia. Rev Panam Salud Publica. 2010; 27(5):352-9.

2. Heininger U, Cherry JD, Stehr K, Schmitt-Grohe S, Uberall M, Laussucq S, et al. Comparative Efficacy of the Lederle/Takeda acellular pertussis component DTP (DTaP) vaccine and Lederle whole-cell component DTP vaccine in German children after household exposure. Pertussis Vaccine Study Group. Pediatrics. 1998;102(3 Pt 1):546-53.

3. Greco D, Salmaso S, Mastrantonio P, Giuliano M, Tozzi AE, Anemona A, et al. A controlled trial of two acellular vaccines and one whole-cell vaccine against pertussis. Progetto Pertosse Working Group. N Engl J Med. 1996;334(6):341-8.

4. Bisgard KM, Rhodes P, Connelly BL, Bi D, Hahn C, Patrick S, et al. Pertussis vaccine effectiveness among children 6 to 59 months of age in the United States, 1998-2001. Pediatrics. 2005; 116(2):e285-94.

5. Ekwueme DU, Strebel PM, Hadler SC, Meltzer MI, Allen JW, Livengood JR. Economic evaluation of use of diphtheria, tetanus, and acellular pertussis vaccine or diphtheria, tetanus, and whole-cell pertussis vaccine in the United States, 1997. Arch Pediatr Adolesc Med. 2000;154(8):797-803.

6. Le Saux N, Barrowman NJ, Moore DL, Whiting S, Scheifele D, Halperin S. Decrease in hospital admissions for febrile seizures and reports of hypotonic-hyporesponsive episodes presenting to hospital emergency departments since switching to acellular pertussis vaccine in Canada: a report from IMPACT. Pediatrics. 2003;112(5):e348. 
7. Mascareñas A, Salinas J, Tasset-Tisseau A, Mascareñas C, Khan MM. Polio immunization policy in Mexico: economic assessment of current practice and future alternatives. Public Health. 2005;119(6):542-9.

8. Khan MM. Economics of polio vaccination in the posteradication era: should OPV-using countries adopt IPV? Vaccine. 2008;26(16):2034-40

9. Barbieri M, Drummond M, Rutten F, Cook J, Glick HA, Lis J, et al.; ISPOR Good Research Practices Economic Data Transferability Task Force. What do international pharmacoeconomic guidelines say about economic data transferability? Value Health. 2010;13(8):1028-37.

10. Turk F. Data generalizability, data transferability, and the political economy of pharmacoeconomic guidelines. Value Health. 2010;13(8):863-4.

11. Drummond M, Barbieri M, Cook J, Glick HA, Lis J, Malik F, et al. Transferability of economic evaluations across jurisdic- tions: ISPOR Good Research Practices Task Force report. Value Health. 2009;12(4):409-18.

12. Augustovski F, Iglesias C, Manca A, Drummond M, Rubinstein A, Martí SG. Barriers to generalizability of health economic evaluations in Latin America and the Caribbean region. Pharmacoeconomics. 2009;27(11):919-29.

13. Miller MA, Sutter RW, Strebel PM, Hadler SC. Cost-effectiveness of incorporating inactivated poliovirus vaccine into the routine childhood immunization schedule. JAMA. 1996; 276(12):967-71.

14. Tucker AW, Isaacs D, Burgess M. Cost-effectiveness analysis of changing from live oral poliovirus vaccine to inactivated poliovirus vaccine in Australia. Aust N Z J Public Health. 2001;25(5):411-6.

15. Griffiths UK, Botham L, Schoub BD. The cost-effectiveness of alternative polio immunization policies in South Africa. Vaccine. 2006;24(29-30):5670-8. 